\title{
All-dielectric meta-holograms with holographic images transforming longitudinally
}

Qiu Wang, Quan Xu, Xueqian Zhang, Chunxiu Tian, Yuehong Xu, Jianqiang Gu, Zhen Tian, Chunmei Ouyang, Xixiang Zhang, Jiaguang Han, and Weili Zhang

ACS Photonics, Just Accepted Manuscript • DOI: 10.1021/acsphotonics.7b01173 • Publication Date (Web): 22 Nov 2017

Downloaded from http://pubs.acs.org on December 3, 2017

\section{Just Accepted}

"Just Accepted" manuscripts have been peer-reviewed and accepted for publication. They are posted online prior to technical editing, formatting for publication and author proofing. The American Chemical Society provides "Just Accepted" as a free service to the research community to expedite the dissemination of scientific material as soon as possible after acceptance. "Just Accepted" manuscripts appear in full in PDF format accompanied by an HTML abstract. "Just Accepted" manuscripts have been fully peer reviewed, but should not be considered the official version of record. They are accessible to all readers and citable by the Digital Object Identifier (DOI®). "Just Accepted" is an optional service offered to authors. Therefore, the "Just Accepted" Web site may not include all articles that will be published in the journal. After a manuscript is technically edited and formatted, it will be removed from the "Just Accepted" Web site and published as an ASAP article. Note that technical editing may introduce minor changes to the manuscript text and/or graphics which could affect content, and all legal disclaimers and ethical guidelines that apply to the journal pertain. ACS cannot be held responsible for errors or consequences arising from the use of information contained in these "Just Accepted" manuscripts. 


\title{
All-dielectric meta-holograms with holographic images transforming longitudinally
}

Qiu Wang ${ }^{\dagger}$, Quan Xu $\mathrm{Xu}^{\dagger}$, Xueqian Zhang ${ }^{\dagger}$, Chunxiu Tian ${ }^{\dagger}$, Yuehong $\mathrm{Xu}^{\dagger}$, Jianqiang $\mathrm{Gu}^{\dagger}$, Zhen Tian $^{\dagger}$, Chunmei Ouyang ${ }^{\dagger}$, Xixiang Zhang ${ }^{*}, \dot{*}$, Jiaguang Han ${ }^{*}, \dagger$, and Weili Zhang ${ }^{*}, \dagger, \S$

${ }^{\dagger}$ Center for Terahertz waves and College of Precision Instrument and Optoelectronics Engineering, Tianjin University and the Key Laboratory of Optoelectronics Information and Technology (Ministry of Education), Tianjin 300072, China.

${ }^{*}$ Physical Science and Engineering Division, King Abdullah University of Science and Technology, Thuwal 23955-6900, Saudi Arabia.

${ }^{\S}$ School of Electrical and Computer Engineering, Oklahoma State University, Stillwater, Oklahoma 74078, USA.

\begin{abstract}
Metasurfaces are unique subwavelength geometries capable of engineering electromagnetic waves at will, delivering new opportunities for holography. Most previous meta-holograms, so-called phase-only meta-holograms, modulate only the amplitude distribution of a virtual object, and require optimizing techniques to improve the image quality. However, the phase distribution of the reconstructed image is usually overlooked in previous studies, leading to inevitable information loss. Here, we demonstrate all-dielectric meta-holograms that allow tailoring of both the phase and amplitude distributions of virtual objects. Several longitudinal manipulations of the holographic images are theoretically and experimentally demonstrated, including shifting, stretching, and rotating, enabling a large depth of focus. Furthermore, a new meta-hologram with a three-dimensional holographic design method is demonstrated with an even enhanced depth of focus. The proposed meta-holograms offer more freedom in holographic design and open new avenues for designing complex three-dimensional holography.
\end{abstract}


KEY WORDS: meta-hologram, all-dielectric metasurface, phase-amplitude modulation, three-dimensional, terahertz

\section{TOC GRAPHIC}

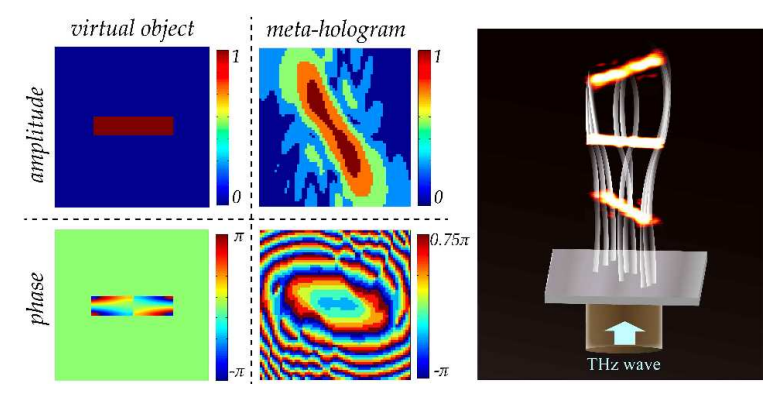


Holograms record the complete amplitude and phase information of light scattering by an object, thus capturing a three-dimensional (3D) image to project. Holography is originally proposed by Gabor in $1948 .{ }^{1}$ As computer technology and optoelectronics rapidly advance, computer-generated holography (CGH) emerges, where holograms are created by numerical calculation and reconstructed optically. ${ }^{2} \mathrm{CGH}$ has been widely accepted, as it does not require real objects and can be applied across the whole electromagnetic spectrum. Conventional CGH often uses spatial light modulators (SLMs) as holograms. Recently, the adoption of metasurfaces as holograms in $\mathrm{CGH}$ has produced high-resolution meta-holograms and high efficiency meta-holography. ${ }^{3-15}$

Metasurfaces, which typically consist of a monolayer of subwavelength plasmonic or dielectric structures, are simple to fabricate, exhibit relatively low absorption loss, and have fascinating electromagnetic characteristics. ${ }^{16,17}$ Metasurfaces can be designed to control the polarization, phase, and amplitude of outgoing electromagnetic waves as desired, providing an exciting platform for ultrathin optics. Ultrathin metasurfaces have much smaller unit cell sizes and thicknesses than their conventional counterparts. Thanks to these unique properties, metasurfaces have achieved various intriguing phenomena and applications, such as ultrathin flat lenses, ${ }^{18-22}$ anomalous reflection and refraction, ${ }^{23-26}$ optical vortex generation, ${ }^{27-29}$ wave plates, ${ }^{30,31}$ and meta-holography. ${ }^{3-15}$ Plasmonic metasurfaces easily support electromagnetic resonances; however, they typically suffer from low efficiency due to ohmic loss. Composed of high refractive index resonators, dielectric metamaterial unit cells use Mie resonances to generate electric and magnetic resonances with dramatically improved efficiency. ${ }^{32,33}$

Most past demonstrations of meta-holography have used phase-only holograms, where 
optimizing techniques such as the Gerchberg-Saxton method were adopted to improve the holographic image quality. ${ }^{34-36}$ However, these optimization techniques cause the phase distribution of the reconstructed image to become irregular and uncontrollable. As amplitude and phase are two fundamental characteristics of electromagnetic waves, the uncontrollable phase distribution clearly constrains the holographic design. Rather than employing optimizing techniques, a new approach could provide simultaneous control of amplitude and phase of each pixel in the metasurface ${ }^{7,37}$ to achieve high quality holographic images. With this approach, both amplitude and phase information of virtual objects can be reconstructed. However, this meta-hologram by adopting single-layer plasmonic resonators ${ }^{7}$ has been difficult to move beyond the limitations in the inherent Ohmic losses and the orthogonal polarization conversion efficiency. A higher efficiency is required in practical applications of the simultaneous amplitude and phase control technique. Also, only amplitude distribution of the virtual object is considered in that work, while the phase distribution is kept constant. This is just like many other studies on meta-holography, where the main concern is the quality of the reconstructed intensity distribution, since the phase information cannot be recorded directly by the eyes or detectors. The effect of engineering both the phase and amplitude distributions of virtual objects in meta-holography has not been further studied yet.

In this article, we propose and demonstrate all-dielectric meta-holograms that continuously transform holographic images along the longitudinal direction by engineering both the phase and amplitude distributions of the virtual objects in the terahertz regime. Dielectric cubic silicon resonators are used as basic unit cells to achieve a simultaneous multi-level modulation of the amplitude and phase distributions on the holograms with 
relatively high efficiency. Fiber-based near-field scanning terahertz microscopy (FNSTM) is employed in the experimental measurements with a high scanning speed and high resolution. The shifting, stretching, and rotating of the images along the direction of electromagnetic wave propagation are theoretically and experimentally demonstrated, and indicate a large depth of focus (DOF). The DOF, that illustrates the tolerance of a hologram in the position where an image is detected, is a very important parameter and reflects the longitudinal resolution. The DOF of our hologram is proven to be larger than that of the phase-only hologram with optimization. Moreover, a new design approach for three dimensional (3D) holograms is proposed with further enhanced DOF. The proposed meta-holograms allow more freedom in the holographic design process through engineering both of the phase and amplitude distributions of the virtual 3D objects. The larger DOF accelerates the pace of meta-holography towards practical applications.

\section{DESIGN}

Here, subwavelength silicon pillar resonators are employed as basic unit cells, as shown in Figure 1a. The pillars are made from $150 \mu \mathrm{m}$ thick high-resistivity silicon and patterned on a $2 \mathrm{~mm}$ thick high-resistivity silicon substrate. According to the effective media theory, a large effective refractive index leads to a slow electromagnetic wave speed, whose direction of propagation can be defined as the slow axis and the fast axis is thus along the orthogonal direction, as illustrated in Figure $1 \mathrm{~b}$. When the optical path difference between the two polarized components along the slow axis and the fast axis is equal to half of a wavelength, 
the linearly polarized incidence converts nearly completely into the orthogonal polarized component, where the silicon pillar serves as a half-waveplate.

The performance of the all-dielectric meta-holography is numerically simulated using a commercial software, CST Microwave Studio. Four silicon pillars with approximately 0.9 amplitude transmission and a $\pi / 4$ phase-shift interval of the outgoing $y$-polarized electric field components $E_{y}$ are selected under $1.0 \mathrm{THz}$ and an $x$-polarized incident plane wave. Their geometric parameters are $(a, b)=(48 \mu \mathrm{m}, 115 \mu \mathrm{m}),(40 \mu \mathrm{m}, 112 \mu \mathrm{m}),(35 \mu \mathrm{m}, 93 \mu \mathrm{m})$, and $(24 \mu \mathrm{m}, 101.5 \mu \mathrm{m})$, respectively. By incorporating the additional $x$-axis-mirrored unit cells, eight silicon pillar resonators are obtained, composing a phase shift ranging from zero to $2 \pi$ and nearly the same transmissions, as shown in Figure 1c. All eight silicon pillars are designed with same $(P, h)=(130 \mu \mathrm{m}, 150 \mu \mathrm{m})$ for ease of fabrication. Based on the coordinate system transformation theory, when a silicon pillar rotates around the $z$-axis, the phase shift of the outgoing $y$-polarized component remains constant under an $x$-polarized incidence, except for a $\pi$ jump when the slow axis or the fast axis passes the $x$-axis. Meanwhile, the amplitude of the $y$-polarized electric field component $\left|E_{y}\right|$ is proportional to $|\sin (2 \theta)|$, where $\theta$ represents the orientation of the slow axis relative to the $x$-axis. As $\theta$ varies from $-90^{\circ}$ to $90^{\circ}$ in a $5^{\circ}$ interval, the numerically simulated results of the amplitude and phase shifts of the outgoing $y$-polarized component $E_{y}$ for the first silicon pillar of the eight unit cells remain consistent with our expectations. Thus, the simultaneous modulation of phase and amplitude in each pixel of the metasurface has been achieved with high efficiency. 
RESULTS

\begin{abstract}
Most of the meta-holograms demonstrated before only focus on the amplitude distributions of holographic images. Many optimizing techniques, including the Gerchberg-Saxton method, are employed in these phase-only holograms to improve the image quality; however, the trade-off is that the phase distribution becomes uncontrollable. Here, by elaborately engineering both the phase and amplitude distributions of two-dimensional (2D) virtual objects, several manipulations of the holographic images along the longitudinal direction (z-axis), namely different image transformations in 3D space are achieved as presented in detail below.
\end{abstract}

\title{
Longitudinal manipulations of the holographic images: shifting
}

An image of the letter "T" with a constant amplitude is designed to appear in the plane $z$ $=6 \mathrm{~mm}$. The size of the observed region is $8 \times 8 \mathrm{~mm}^{2}$, as shown in Figure 2a. The horizontal and vertical rectangles of the " $\mathrm{T}$ " are designed to move in opposite directions along the $x$-axis. Thus, the normalized electric field distribution of the virtual object is

$$
. E(x, y)=\left\{\begin{array}{cc}
\exp \left(i k_{1} x\right), & x \in[-2,2], y \in[1.5,2.5] \\
\exp \left(-i k_{1} x\right), & x \in[-0.5,0.5], y \in[-2.5,1.5) \\
0, & \text { else }
\end{array}\right.
$$

Here, we let $k_{1}=2 \pi \mathrm{rad} / \mathrm{mm}$. The electric field phase distribution of the virtual object is shown in Figure $2 b$.

To design the corresponding meta-hologram, the Rayleigh-Sommerfeld diffraction theory is applied. After normalization, the linear partition method is employed to discretize the amplitude into five levels $(0,0.25,0.5,0.75$, or 1$)$. By utilizing silicon pillar resonators as 
the basic unit pixels, the simultaneous eight-level phase and five-level amplitude modulation is achieved. The electric field amplitude and phase distributions of the meta-hologram can be seen in Figure $2 \mathrm{c}$ and $\mathrm{d}$, respectively. Composed by $61 \times 61$ pixels, the dielectric meta-hologram is fabricated by conventional deep reactive ion etching (DRIE), whose partial scanning electron microscopy image is shown in Figure 2e. Thus, the size of the meta-hologram is also approximately $8 \times 8 \mathrm{~mm}^{2}$.

The Rayleigh-Sommerfeld diffraction theory is applied again in the image reconstruction. The numerically simulated electric field amplitude distributions $\left|E_{y}\right|$ under 1.0 $\mathrm{THz}$ at different locations ranging from $z=4$ to $8 \mathrm{~mm}$ are shown in Figure $2 \mathrm{f}$. The " $\mathrm{T}$ " image appears at $z=6 \mathrm{~mm}$, and then changes into " $\Gamma$ " and " $\urcorner$ " at $z=4 \mathrm{~mm}$ and $z=8 \mathrm{~mm}$, respectively.

FNSTM is employed in the experimental demonstrations. The measured $y$-polarized electric field amplitude distributions $\left|E_{y}\right|$ under $1.0 \mathrm{THz}$ and $x$-polarized illumination at different locations along the $z$-axis are shown in Figure $2 \mathrm{~g}$. The electric field is detected at $0.25 \mathrm{~mm}$ intervals from -4 to $4 \mathrm{~mm}$ in both the $x$ and $y$ directions, and at a $1 \mathrm{~mm}$ interval from 4 to $8 \mathrm{~mm}$ in the $z$ direction. The shifting of the two "T" components along the $z$-axis can be clearly seen with good image quality from $z=4$ to $8 \mathrm{~mm}$, which coincides well with the simulated results in terms of image size, profile, location, and relative amplitude distribution, demonstrating good control over shifting in the meta-hologram. There's a little quality difference between the reconstructed vertical rectangle and the reconstructed horizontal rectangle, especially in the edges of these rectangles, which is mainly due to the information loss caused by the limited hologram size. As can be seen in Figure 2c, the right edge of the 


\section{Longitudinal manipulations of the holographic images: stretching}

The methods for meta-hologram design, sample fabrication, numerical simulation, and experimental measurements are the same as in the shifting exercise. The same " $T$ " with a constant amplitude is designed to appear in the plane $z=6 \mathrm{~mm}$, as shown in Figure 3a. The image is designed to stretch along the $z$-axis, so the normalized electric field distribution of

the virtual object is

horizontal rectangular region in the hologram is "cut off" by the boundary of the hologram, while the edges of the vertical one are overall exhibited in the hologram. The missing information will mainly affect its corresponding image region, considering that the image plane is just $6 \mathrm{~mm}$ away from the hologram (twentyfold wavelength for $1 \mathrm{THz}$ ). That's why the main difference appears in the edges of the rectangles and why the left edge of the horizontal rectangle exhibits better than the right edge. The quality of the reconstructed horizontal rectangle will be better if the hologram is large enough to contain all the amplitude-not-zero pixels. Besides, the speed of the image shifting can be easily manipulated by varying $k_{1}$. The measured transmission efficiency, defined as the ratio between the measured $y$-polarized terahertz power in the " $T$ " region at $z=6 \mathrm{~mm}$ and the input power, reaches $16.3 \%$ when employing the dielectric silicon pillar resonators. The corresponding simulated transmission efficiency of the silicon pillars is $18.8 \%$, which is much higher than that of the single-layer transmission-type plasmonic structures with simultaneous phase and amplitude modulation $(6.4 \%$ in our previous work $){ }^{7}$ The good holographic image quality and the high efficiency promise practical applications in meta-holography. 


$$
E(x, y)=\left\{\begin{array}{cc}
\exp \left(i k_{2}|x|\right), & x \in[-2,2], y \in[1.5,2.5] \\
\exp \left[i k_{2}(2-y)\right], & x \in[-0.5,0.5], y \in[-2.5,1.5) \\
0, & \text { else }
\end{array}\right.
$$

Here, we let $k_{2}=\pi \mathrm{rad} / \mathrm{mm}$. The electric field phase distribution of the virtual object is shown in Figure 3b.

The electric field amplitude and phase distributions of the meta-hologram are exhibited in Figure 3c and d, where the eight-level phase and five-level amplitude modulations are also employed. The simulated and measured amplitude distributions of the $y$-polarized electric field component under an $x$-polarized normal incidence at different locations along the $z$-axis are shown in Figure $3 \mathrm{f}$ and $\mathrm{g}$. The simulated and measured results are in good agreement, exhibiting a stretching effect along the $z$-axis with a range from $z=4$ to $8 \mathrm{~mm}$. The holographic image " $\mathrm{T}$ " becomes smaller as the distance from the meta-hologram increases, which is the opposite of conventional optical imaging behavior. The simulated magnification between the image profiles at planes $z=4 \mathrm{~mm}$ and $z=8 \mathrm{~mm}$ is 1.33 along the $x$ direction and 1.17 along the $y$ direction, and the measured magnification is 1.38 and 1.10 , respectively. The magnification can be easily controlled by changing $k_{2}$. The measured and simulated efficiencies for this meta-hologram are $14.5 \%$ and $19.6 \%$, respectively. Note that for the shifting and stretching manipulations, the virtual objects are designed with the same amplitude distributions (the letter "T") and different phase distributions. Completely different longitudinal exhibitions of holographic images are achieved, which overall exhibit different 3D distributions, indicating the functionality of incorporating phase control into the virtual object. 


\section{Longitudinal manipulations of the holographic images: rotating}

The virtual object in this experiment is a rectangle with a constant amplitude in the plane $z=6 \mathrm{~mm}$, as shown in Figure 4a. The rectangle is designed to be capable of rotating along the $z$-axis, and every point should have the same space angular velocity $\omega_{0}$ for image stability. Under the cylindrical coordinate system, when a point $(r, \theta, z)$ moves to its next location $(r+\mathrm{d} r, \theta+\mathrm{d} \theta, z+\mathrm{d} z)$, we know that $v_{r} / c=k_{r} / k$. Here $v_{r}$ and $k_{r}$ represent the space linear velocity component and the wave vector component in the $r \theta$-plane, respectively; $c$ and $k$ are the velocity of light and wave vector in vacuum, respectively; $k_{r}=\mathrm{d} \varphi / \mathrm{d} l$, where $\mathrm{d} \varphi$ and $\mathrm{d} l$ are differential phase shift and distance between the two points, respectively. So we can get $v_{r}=$ $c \mathrm{~d} \varphi / k \mathrm{~d} l$. Since $v_{r}=\omega_{0} r$ and $\mathrm{d} l=r \mathrm{~d} \theta$, we can obtain $\mathrm{d} \varphi=\omega r^{2} \mathrm{~d} \theta$, where $\omega=\omega_{0} k / c$ is a constant. After integration, the final phase distribution can be obtained as $\varphi=\omega r^{2} \theta$. Thus, the normalized electric field distribution of the virtual object is

$$
E(x, y)=\left\{\begin{array}{ccc}
\exp \left[i \omega\left(x^{2}+y^{2}\right) \arctan \frac{y}{x}\right], & x \in[-2,2], y \in[-0.4,0.4] & \text { (mm) } \\
0, & \text { else }
\end{array}\right.
$$

Here, we let $\omega=4.5 \mathrm{~mm}^{-2}$. The electric field phase distribution of the virtual object is shown in Figure 4b.

Similarly, the electric field amplitude and phase distributions of the meta-hologram, partial scanning electron microscopy image, and the simulated and measured electric field amplitude distributions are shown in Figure 4c-g. Satisfactory rotating is observed, where the simulated rotation angle of the profile of the rectangle from $z=3$ to $9 \mathrm{~mm}$ is $79^{\circ}$, and the measured result is $78^{\circ}$. The rotation angle can be controlled by varying $\omega$. The measured electric field amplitude distributions are in good coincidence with the simulations, including 
the image size, profile, orientation angle, and relative amplitude distribution, which demonstrates the good rotating manipulation of the holographic images. In addition, the measured and simulated efficiencies for this meta-hologram are $8.6 \%$ and $11.6 \%$, respectively.

\section{Meta-holography with a large DOF}

Our results indicate that a large DOF can be obtained with the proposed holographic design approach without the use of optimizing techniques. Irregular phase distribution from optimizing techniques leads to poor directivity along the propagation direction, which would also reduce the DOF. To further investigate the DOF of different meta-holograms, we design three meta-holograms. One is a phase-only meta-hologram optimized by the Gerchberg-Saxton method. The second is the meta-hologram of our design with simultaneous eight-level phase and five-level amplitude modulation. Each of the two meta-holograms is designed to reconstruct a $2 \mathrm{D}$ virtual object with a constant amplitude distribution " $\mathrm{T}$ " located at $z=6 \mathrm{~mm}$, which is the same with Figure 2a. A constant phase distribution of the 2D virtual object is designed for the second meta-hologram with simultaneous phase and amplitude modulation; however, the 2D virtual object's phase distribution of the phase-only hologram is uncontrollable and unable to be designed. Third, we propose 3D holographic design approach to achieve an even larger DOF, where the virtual object is a $3 \mathrm{D}$ " $\mathrm{T}$ " profile from $z=2$ to 10 $\mathrm{mm}$ with a constant amplitude, as shown in Figure 5a. The phase distribution is a constant in each $x y$-plane, but a $k \times z$ phase shift is designed along the $z$-axis, as shown in Figure $5 \mathrm{~b}$. Here, we let $k$ equal the wave vector in an electromagnetic wave vacuum under $1.0 \mathrm{THz}$. The 
Rayleigh-Sommerfeld diffraction theory is used and by sum of the contributions of all $x y$-planes from $z=2$ to $10 \mathrm{~mm}$, the electric field distribution in this $3 \mathrm{D}$ designed meta-hologram is obtained. Simultaneous eight-level phase and five-level amplitude modulation is employed to construct this $3 \mathrm{D}$ designed meta-hologram.

The measured electric field amplitude distributions of the three meta-holograms under 1.0 THz are shown in Figure 6a-m. In the results of the phase-only meta-hologram, the "T" begins to "split" at $z=5 \mathrm{~mm}$ and to "granulate" at $z=7 \mathrm{~mm}$. In both the meta-hologram with simultaneous phase and amplitude modulation and the 3D designed meta-hologram, the image quality along the $z$-axis is extensively enhanced. The 3D designed meta-hologram shows even better imaging results than the other two meta-holograms at $z=2 \mathrm{~mm}$ and $z=10$ $\mathrm{mm}$ in particular, demonstrating the enhanced DOF of the 3D holographic design approach. The different DOF character of these three meta-holograms can be more clearly seen in the simulated holographic imaging results shown in Figure S1 (See Supplementary Note 1). The measured efficiencies of the phase-only meta-hologram, the meta-hologram with simultaneous phase and amplitude modulation, and the 3D designed meta-hologram are $44.5 \%, 13.7 \%$, and $13.3 \%$, respectively. Their corresponding simulated efficiencies are $65.1 \%, 17.2 \%$, and $15.4 \%$, respectively.

Given the Strehl ratio in conventional optics, the DOF in holography can be defined as the longitudinal range of the image region in which the integral intensity is more than $80 \%$ of the integral intensity in the designed focal plane. Experimentally, it is time-consuming and extremely difficult to measure the precise DOF as scanning detection is adopted in the $x y$-plane, which is quite different from the charge coupled device (CCD) detection method 
used in the visible regime. Therefore, numerical simulations are employed for the quantitative analysis of DOF. The simulated results for the DOF are $2.8 \mathrm{~mm}$ (from $z=5.3$ to $8.1 \mathrm{~mm}$ ) for the phase-only meta-hologram, $5.0 \mathrm{~mm}$ (from $z=3.8$ to $8.8 \mathrm{~mm}$ ) for the meta-hologram, and $7.9 \mathrm{~mm}$ (from $z=2.7$ to $10.6 \mathrm{~mm}$ ) for the 3D designed meta-hologram. The simulated and measured results demonstrate that the meta-hologram with simultaneous phase and amplitude modulation has a larger DOF than the phase-only meta-hologram, and that the $3 \mathrm{D}$ designed meta-hologram exhibits even further improvement. In addition, the DOF is directly proportional to the object distance and inversely proportional to the aperture. Considering the small object distance $(6 \mathrm{~mm})$ and the large size of the meta-hologram $\left(8 \times 8 \mathrm{~mm}^{2}\right)$, it is very exciting to realize a $7.9 \mathrm{~mm}$ DOF with the 3D design approach. Regarding to the large DOF, we emphasize here that it is very helpful to obtain good images in a long range, which can make the hologram become more robust in real applications. However, for some other 3D holograms, such as irrelevant images at different plane, the large DOF may become crosstalk at other imaging plane in a sense. In that case, one needs other methods to optimize the phase distribution of each virtual image to restrain the crosstalk. By combining the iterative multi-plane holography algorithm with our design method, it might be possible to suppress the crosstalk among different imaging planes if we elaborately design proper initial phase distributions of the multi-plane images to make them capable of propagating approximately from one to the next.

\section{CONCLUSION}


We demonstrate a new meta-hologram design method by engineering both the phase and amplitude distributions of a virtual object. Relatively high efficiency is obtained by employing dielectric silicon pillar resonators as the basic unit pixels. The shifting, stretching, and rotating of holographic images along the longitudinal direction (z-axis) are demonstrated both theoretically and experimentally, indicating the additional freedom in our holographic design approach and suggesting further manipulation possibilities. The clear holographic images along the propagation direction also indicate a large DOF. A comparison of the DOF of a phase-only meta-hologram, a meta-hologram with simultaneous amplitude and phase control, and a new meta-hologram with 3D holographic design approach confirm that the 3D designed meta-hologram has a much larger DOF than other meta-holograms.

Compared with the phase-only meta-holograms that achieve multi-plane images or 3D images, our both phase and amplitude modulated meta-holograms by engineering both the phase and amplitude distributions of a virtual object can uniquely achieve holographic images continuously transforming along the longitudinal direction in a long range with even no crosstalk. Besides, our design approach is direct and the algorithm is simple and timesaving. Furthermore, from the perspective of information theory, the meta-holograms with both phase and amplitude modulation contain more degree of freedom and thus, contain more information. Hence, they can be designed to realize more complex and flexible holographic images. It is believed that the proposed holographic design approach provides a new method for 3D holographic imaging and has many prospects in practical applications.

\section{METHODS}




\begin{abstract}
Sample fabrication. All the meta-holograms are fabricated by using conventional lithography together with deep reactive ion etching. First, the $2 \mathrm{~mm}$ thick double-side polished high-resistivity silicon wafer is sputtered with a $10 \mathrm{~nm}$ thick chromium layer and then spin-coated with a $7 \mu \mathrm{m}$ thick layer of photoresist (AZ2070). Next, by conventional photolithography, the left photoresist and the chromium become a double layer of protection above the silicon pillars. Then, conventional deep reactive ion etching is employed to make the silicon pillars with $150 \mu \mathrm{m}$ thickness. Finally, the remaining photoresist and the remaining chromium are cleaned off separately.
\end{abstract}

Experimental Characterization. FNSTM is employed in the experimental demonstrations for its high scanning speed and high resolution. Detailed description of FNSTM can be found in our previous work. ${ }^{38}$ Here, only the $y$-polarized electric field component is measured with an $x$-polarized illumination. The electric field is detected at $0.25 \mathrm{~mm}$ intervals from -4 to 4 $\mathrm{mm}$ in both the $x$ and $y$ directions. It is further detected at a $1 \mathrm{~mm}$ interval for longitudinal manipulation measurements and a $2 \mathrm{~mm}$ interval for DOF characterizations in the $z$ direction.

\author{
ASSOCIATE CONTENT \\ Supporting Information \\ 1. Simulated results of the three meta-holograms with different DOF.
}

\begin{abstract}
AUTHOR INFORMATION
Corresponding Authors

*Email: jiaghan@tju.edu.cn.

*Email: xixiang.zhang@kaust.edu.sa.

*Email: weili.zhang@,okstate.edu.
\end{abstract}




\section{Notes}

The authors declare no competing financial interest.

\section{ACKNOWLEDGEMENT}

This work was supported by the National Natural Science Foundation of China (Grant Nos. 61422509, 61735012, 61427814, 61420106006, and 61605143), the National Key Basic Research Special Foundation of China (Grant No. 2014CB339800), the Program for Changjiang Scholars and Innovative Research Team in Universities (Grant No. IRT13033), and the U. S. National Science Foundation (Grant No. ECCS-1232081).

\section{REFERENCE}

(1) Gabor, D. A New Microscopic Principle. Nature 1948, 161, 777-778.

(2) Lohmann, A. W.; Paris, D. P. Binary Fraunhofer Holograms, Generated by Computer. Appl. Optics 1967, 6, 1739-1748.

(3) Mueller, J. P. B.; Rubin, N. A.; Devlin, R. C.; Groever, B.; Capasso, F. Metasurface Polarization Optics: Independent Phase Control of Arbitrary Orthogonal States of Polarization. Phys. Rev. Lett. 2017, 118, 113901.

(4) Xie, Z.; Lei, T.; Si, G.; Wang, X.; Lin, J.; Min, C.; Yuan, X. Meta-Holograms with Full Parameter Control of Wavefront over a $1000 \mathrm{~nm}$ Bandwidth. ACS Photonics 2017, 4, 2158-2164.

(5) Ye, W.; Zeuner, F.; Li, X.; Reineke, B.; He, S.; Qiu, C. W.; Liu, J.; Wang, Y.; Zhang, S.; Zentgraf, T. Spin and Wavelength Multiplexed Nonlinear Metasurface Holography. Nat. Commun. 2016, 7, 11930. 
(6) Li, X.; Chen, L.; Li, Y.; Zhang, X.; Pu, M.; Zhao, Z.; Ma, X.; Wang, Y.; Hong, M.; Luo, X. Multicolor 3D Meta-Holography by Broadband Plasmonic Modulation. Sci. Adv. 2016, 2, e1601102.

(7) Wang, Q.; Zhang, X.; Xu, Y.; Gu, J.; Li, Y.; Tian, Z.; Singh, R.; Zhang, S.; Han, J.; Zhang, W. Broadband Metasurface Holograms: toward Complete Phase and Amplitude Engineering. Sci. Rep. 2016, 6, 32867.

(8) Huang, K.; Dong, Z.; Mei, S.; Zhang, L.; Liu, Y.; Liu, H.; Zhu, H.; Teng, J.; Luk'yanchuk, B.; Yang, J. K. W.; Qiu, C. W. Silicon Multi-Meta-Holograms for the Broadband Visible Light. Laser Photonics Rev. 2016, 10, 500-509.

(9) Zhang, L.; Mei, S.; Huang, K.; Qiu, C. W. Advances in Full Control of Electromagnetic Waves with Metasurfaces. Adv. Optical Mater. 2016, 4, 818-833.

(10) Huang, L.; Mühlenbernd, H.; Li, X.; Song, X.; Bai, B.; Wang, Y.; Zentgraf, T. Broadband Hybrid Holographic Multiplexing with Geometric Metasurfaces. Adv. Mater. 2015, 27, 6444-6449.

(11) Zheng, G.; Mühlenbernd, H.; Kenney, M.; Li, G.; Zentgraf, T.; Zhang, S. Metasurface Holograms Reaching 80\% Efficiency. Nat. Nanotechnol. 2015, 10, 308-312.

(12) Huang, Y. W.; Chen, W. T.; Tsai, W. Y.; Wu, P. C.; Wang, C. M.; Sun, G.; Tsai, D. P. Aluminum Plasmonic Multicolor Meta-Hologram. Nano Lett. 2015, 15, 3122-3127.

(13) Chen, W. T.; Yang, K. Y.; Wang, C. M.; Huang, Y. W.; Sun, G.; Chiang, I. D.; Liao, C. Y.; Hsu, W. L.; Lin, H. T.; Sun, S.; Zhou, L.; Liu, A. Q.; Tsai, D. P. High-Efficiency Broadband Meta-Hologram with Polarization-Controlled Dual Images. Nano Lett. 2013, $14,225-230$ 
(14) Ni, X.; Kildishev, A. V.; Shalaev, V. M. Metasurface Holograms for Visible Light. Nat. Commun. 2013, 4, 2807.

(15) Huang, L.; Chen, X.; Mühlenbernd, H.; Zhang, H.; Chen, S.; Bai, B.; Tan, Q.; Jin, G.; Cheah, K. W.; Qiu, C. W.; Li, J.; Zentgraf, T.; Zhang, S. Three-Dimensional Optical Holography Using a Plasmonic Metasurface. Nat. Commun. 2013, 4, 2808.

(16) Kildishev, A. V.; Boltasseva, A.; Shalaev, V. M. Planar Photonics with Metasurfaces. Science 2013, 339, 1232009.

(17) Meinzer, N.; Barnes, W. L.; Hooper, I. R. Plasmonic Meta-Atoms and Metasurfaces Nat. Photon. 2014, 8, 889-898.

(18) Khorasaninejad, M.; Chen, W. T.; Oh, J.; Capasso, F. Super-Dispersive off-Axis Meta-Lenses for Compact High Resolution Spectroscopy. Nano Lett. 2016, 16, $3732-3737$.

(19) Arbabi, A.; Ball, A. J.; Bagheri, M.; Faraon, A. Subwavelength-Thick Lenses with High Numerical Apertures and Large Efficiency based on High-Contrast Transmitarrays Nat. Commun. 2015, 6, 7069.

(20) Wang, Q.; Zhang, X.; Xu, Y.; Tian, Z.; Gu, J.; Yue, W.; Zhang, S.; Han, J.; Zhang, W. A Broadband Metasurface-Based Terahertz Flat-Lens Array. Adv. Optical Mater. 2015, 3, $779-785$.

(21) Wang, Q.; Rogers, E. T. F.; Gholipour, B.; Wang, C. M.; Yuan, G.; Teng, J.; Zheludev, N. I. Optically Reconfigurable Metasurfaces and Photonic Devices based on Phase Change Materials. Nat. Photon. 2016, 10, 60-65. 
(22) Chen, B. H.; Wu, P. C.; Su, V. C.; Lai, Y. C.; Chu, C. H.; Lee, I. C.; Chen, J. W.; Chen, Y. H.; Lan, Y. C.; Kuan, C. H.; Tsai, D. P. GaN Metalens for Pixel-Level Full-Color Routing at Visible Light. Nano Lett. 2017, 17, 6345-6352.

(23) Ni, X.; Emani, N. K.; Kildishev, A. V.; Boltasseva, A.; Shalaev, V. M. Broadband Light Bending with Plasmonic Nanoantennas. Science 2012, 335, 427-427.

(24) Sun, S.; Yang, K. Y.; Wang, C. M.; Juan, T. K.; Chen, W. T.; Liao, C. Y.; He, Q.; Xiao, S.; Kung, W. T.; Guo, G. Y.; Zhou, L.; Tsai, D. P. High-Efficiency Broadband Anomalous Reflection by Gradient Meta-Surfaces. Nano Lett. 2012, 12, 6223-6229.

(25) Grady, N. K.; Heyes, J. E.; Chowdhury, D. R.; Zeng, Y.; Reiten, M. T.; Azad, A. K.; Taylor, A. J.; Dalvit, D. A. R.; Chen, H. T. Terahertz Metamaterials for Linear Polarization Conversion and Anomalous Refraction. Science 2013, 340, 1304-1307.

(26) Zhang, X.; Tian, Z.; Yue, W.; Gu, J.; Zhang, S.; Han, J.; Zhang, W. Broadband Terahertz Wave Deflection based on C-Shape Complex Metamaterials with Phase Discontinuities. Adv. Mater. 2013, 25, 4567-4572.

(27) Yu, N.; Genevet, P.; Kats, M. A.; Aieta, F.; Tetienne, J. P.; Capasso, F.; Gaburro, Z. Light Propagation with Phase Discontinuities: Generalized Laws of Reflection and Refraction. Science 2011, 334, 333-337.

(28) Yang, Y.; Wang, W.; Moitra, P.; Kravchenko, I. I.; Briggs, D. P.; Valentine, J. Dielectric Meta-Reflectarray for Broadband Linear Polarization Conversion and Optical Vortex Generation. Nano Lett. 2014, 14, 1394-1399. 
(29) Huang, L.; Chen, X.; Mühlenbernd, H.; Li, G.; Bai, B.; Tan, Q.; Jin, G.; Zentgraf, T.; Zhang, S. Dispersionless Phase Discontinuities for Controlling Light Propagation. Nano Lett. 2012, 12, 5750-5755.

(30) Lin, D.; Fan, P.; Hasman, E.; Brongersma, M. L. Dielectric Gradient Metasurface Optical Elements. Science 2014, 345, 298-302.

(31) Liu, Z.; Li, Z.; Liu, Z.; Cheng, H.; Liu, W.; Tang, C.; Gu, C.; Li, J.; Chen, H. T.; Chen, S.; Tian, J. Single-Layer Plasmonic Metasurface Half-Wave Plates with Wavelength-Independent Polarization Conversion Angle. ACS Photonics 2017, 4, 2061-2069

(32) Zhao, Q.; Zhou, J.; Zhang, F.; Lippens, D. Mie Resonance-based Dielectric Metamaterials. Mater. Today 2009, 12, 60-69.

(33) Jahani, S.; Jacob, Z. All-Dielectric Metamaterials. Nat. Nanotechnol. 2016, 11, 23-36.

(34) Gerchberg, R. W. A Practical Algorithm for the Determination of the Phase from Image and Diffraction Plane Pictures. Optik 1972, 35, 237-246.

(35) Shimobaba, T.; Ito, T. Random Phase-Free Computer-Generated Hologram. Opt. Express 2015, 23, 9549-9554.

(36) Dorsch, R. G.; Lohmann, A. W.; Sinzinger, S. Fresnel Ping-pong Algorithm for Two-Plane Computer-Generated Hologram Display. Appl. Optics 1994, 33, 869-875.

(37) Liu, L.; Zhang, X.; Kenney, M.; Su, X.; Xu, N.; Ouyang, C.; Shi, Y.; Han, J.; Zhang, W.; Zhang, S. Broadband Metasurfaces with Simultaneous Control of Phase and Amplitude. Adv. Mater. 2014, 26, 5031-5036. 
(38) Wang, Q.; Zhang, X.; Plum, E.; Xu, Q.; Xu, Y.; Zhang, H.; Liao, Y.; Gu, J.; Han, J.; Zhang, W. Polarization and Frequency Multiplexed Terahertz Meta-Holography. Adv. Optical Mater. 2017, 5, 1700277. 


\section{Figure Caption}

a
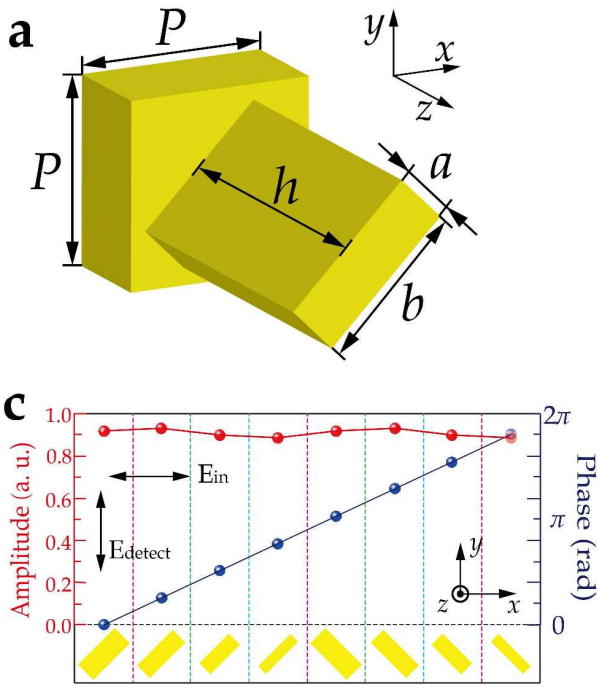

b
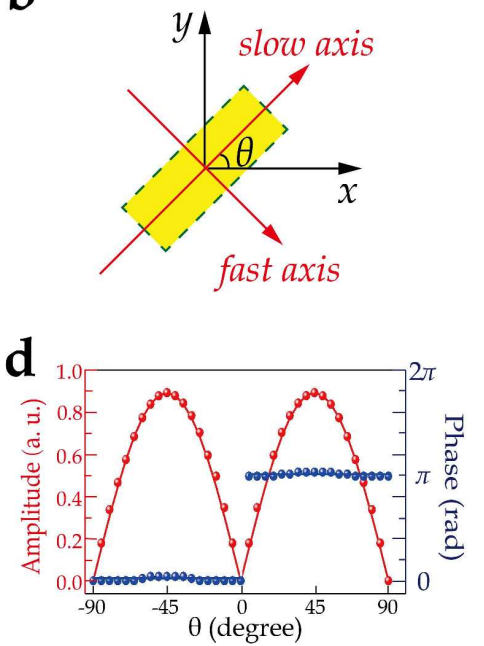

Figure 1. Illustration of the basic functional unit cells. (a) Schematic of a silicon pillar resonator patterned on a silicon substrate. $a, b$, and $h$ represent the width, length, and thickness of the silicon pillar, respectively, and $P$ represents the periodic size of the unit cell. (b) Schematic of the rotation of the silicon pillar. $\theta$ represents the orientation of the slow axis relative to the $x$-axis. (c) Numerically simulated transmission amplitude and phase shift of the $y$-polarized electric field component of the eight unit cells under $1.0 \mathrm{THz}$ and an $x$-polarized normal incidence. (d) The same numerically simulated results as (c) for the first silicon pillar with $(a, b)=(48 \mu \mathrm{m}, 115 \mu \mathrm{m})$, as $\theta$ varies from $-90^{\circ}$ to $90^{\circ}$ in a $5^{\circ}$ interval. The amplitude shift follows a $|\sin (2 \theta)|$ dependence (red solid line), while the phase shift remains constant except for a $\pi$ jump at $\theta=0^{\circ}$. 

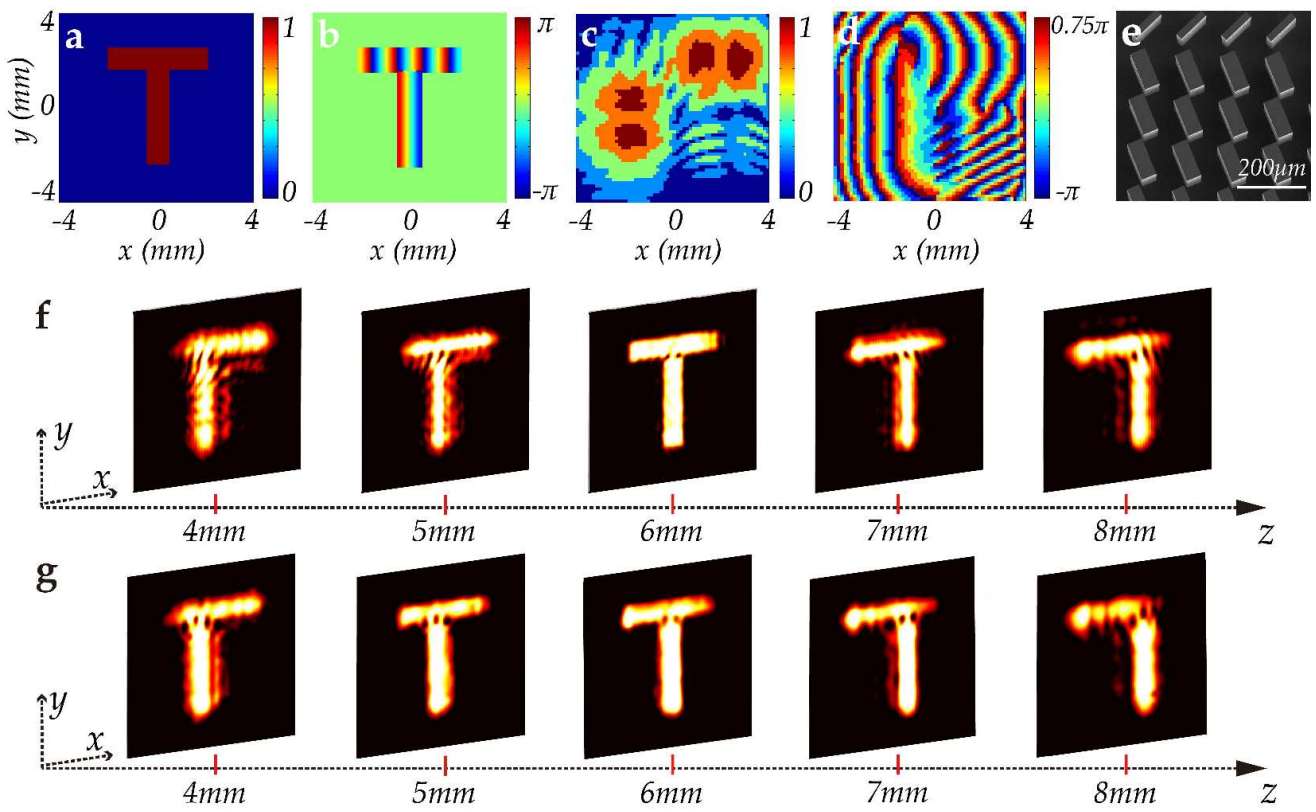

Figure 2. Illustration of the meta-hologram with controlled shifting along the $\boldsymbol{z}$-axis. (a,b) The designed electric field amplitude and phase distributions of the virtual object at $z=6 \mathrm{~mm}$, respectively. (c,d) The designed electric field amplitude and phase distributions of the meta-hologram, respectively, where five-level amplitude and eight-level phase modulation is adopted. (e) Scanning electron microscopy image of the dielectric meta-hologram with shifting manipulation (partial view). (f,g) Simulated and measured amplitude distributions of the $y$-polarized electric field component at $1.0 \mathrm{THz}$ under an $x$-polarized normal incidence at different locations along the $z$-axis. All the observed regions are $8 \times 8 \mathrm{~mm}^{2}$. 

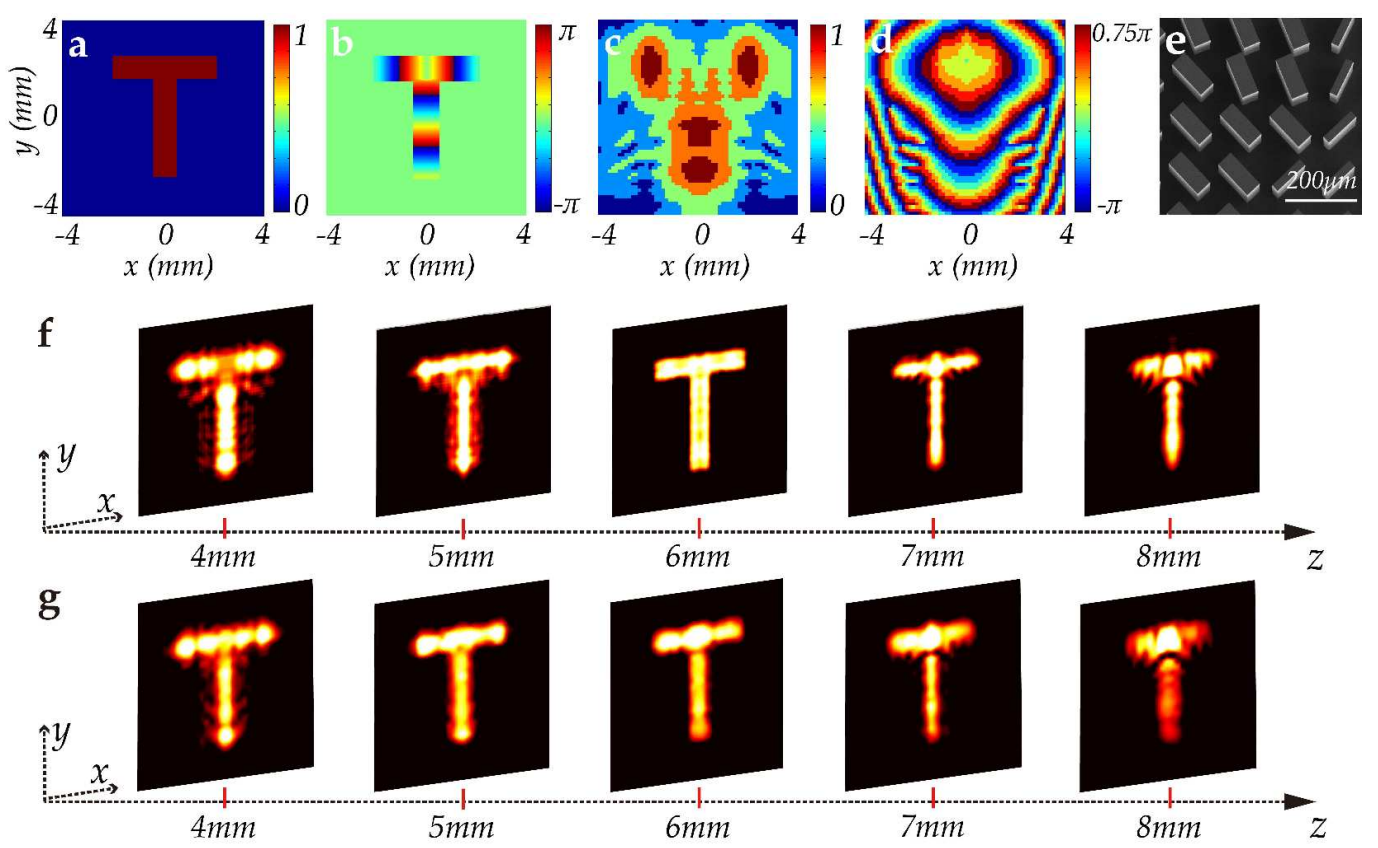

Figure 3. Illustration of the meta-hologram with controlled stretching along the $\boldsymbol{z}$-axis. (a-g) Similar results corresponding to Figure $2 \mathrm{a}-\mathrm{g}$, respectively. 

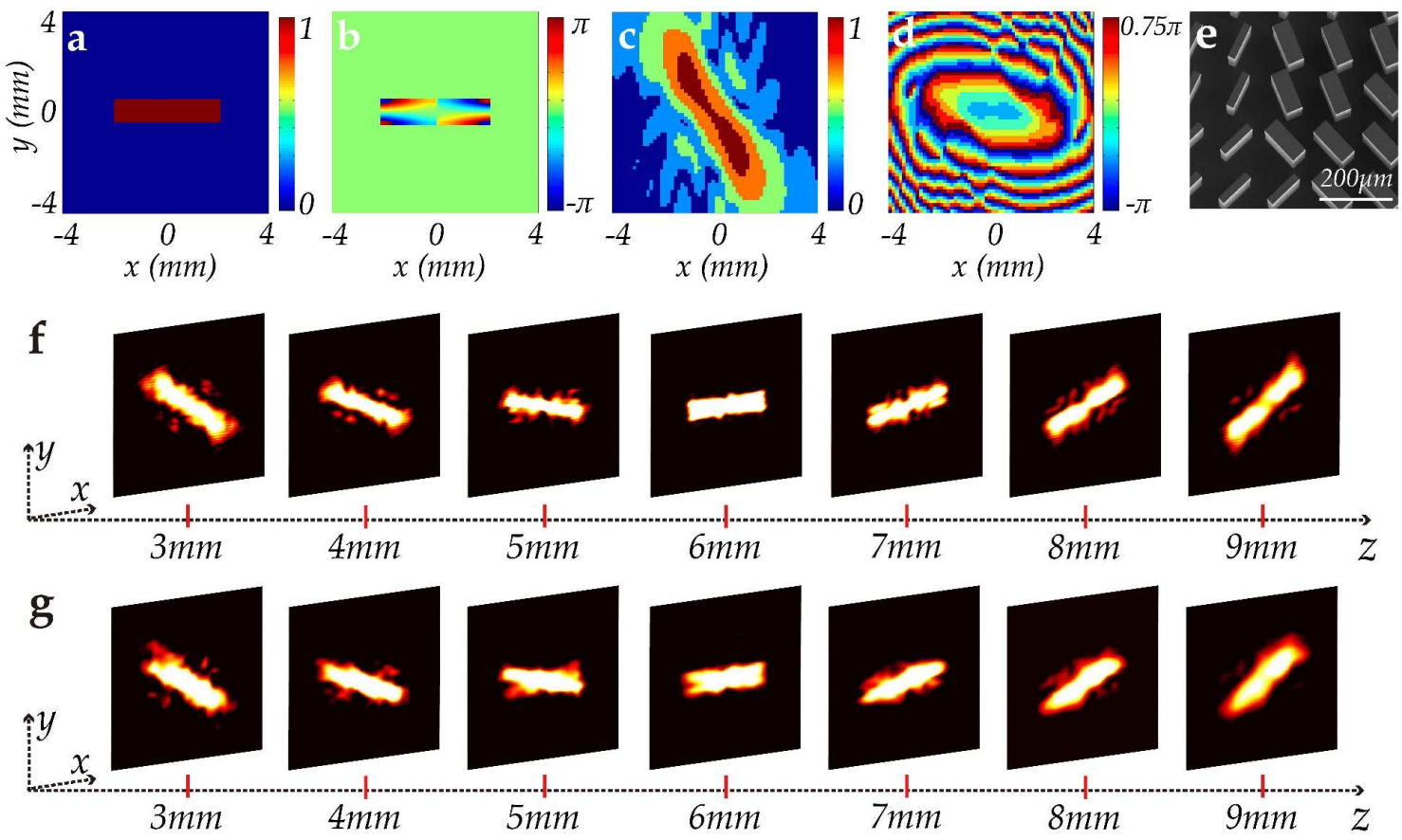

Figure 4. Illustration of the meta-hologram with controlled rotating along the $z$-axis. (a-g) Similar results corresponding to Figure 2a-g, respectively. 

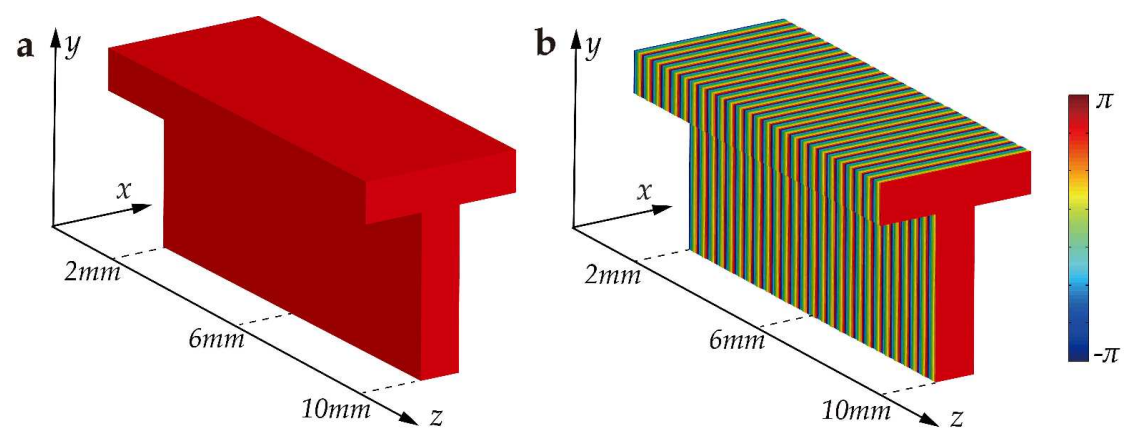

Figure 5. Illustration of the 3D designed meta-hologram. $(a, b)$ The designed electric field amplitude and phase distributions of the 3D virtual object with $z$ ranging from 2 to $10 \mathrm{~mm}$. The sizes along the $x$ and $y$ directions are same as that in Figure 2a. 

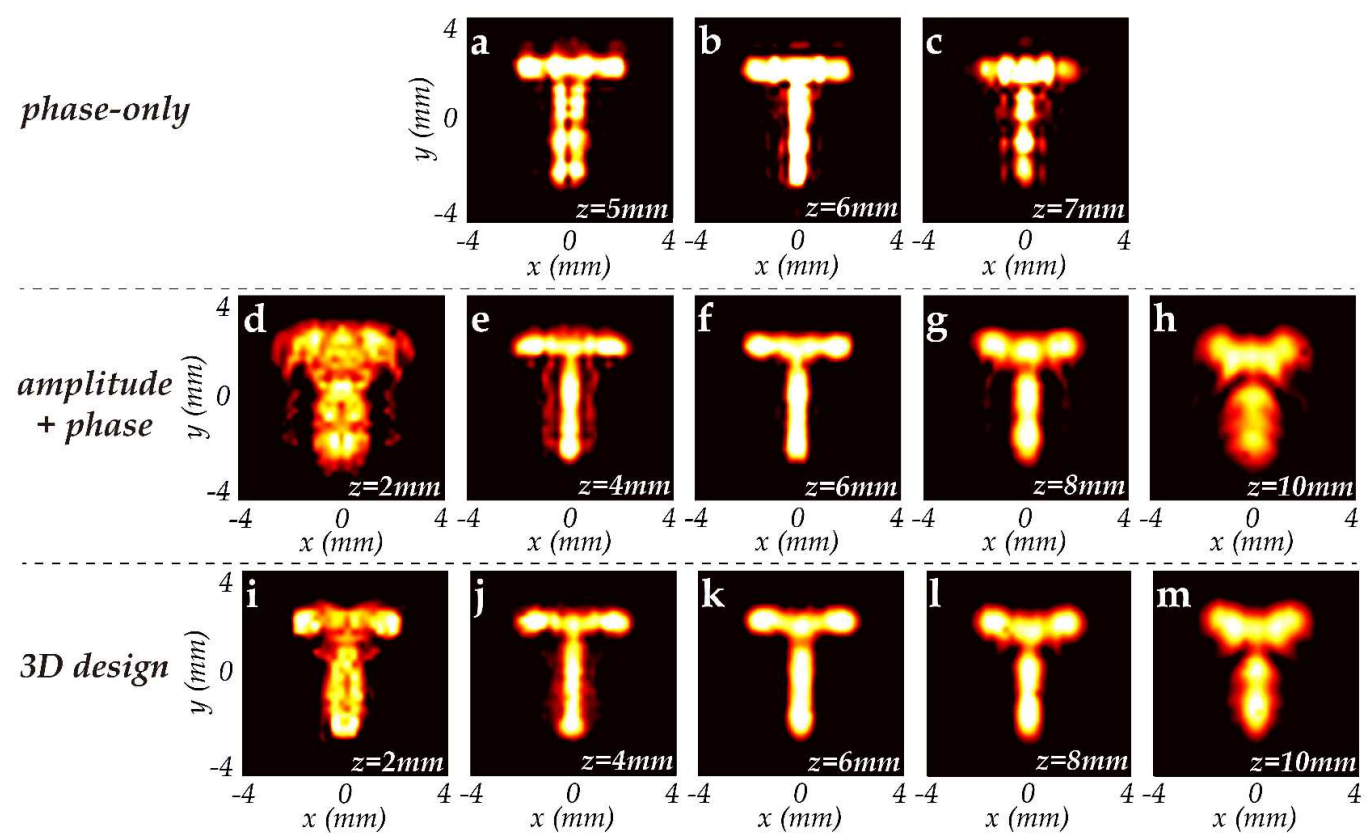

Figure 6. Experimental DOF characterization of the three meta-holograms. Measured electric field amplitude distribution at $1.0 \mathrm{THz}$ at different locations along the $z$-axis, corresponding to (a-c) the phase-only meta-hologram, (d-h) the meta-hologram with simultaneous amplitude and phase control, and (i-m) the 3D designed meta-hologram with simultaneous amplitude and phase control. The location along the $z$-axis is shown at the bottom right corner of each figure. 


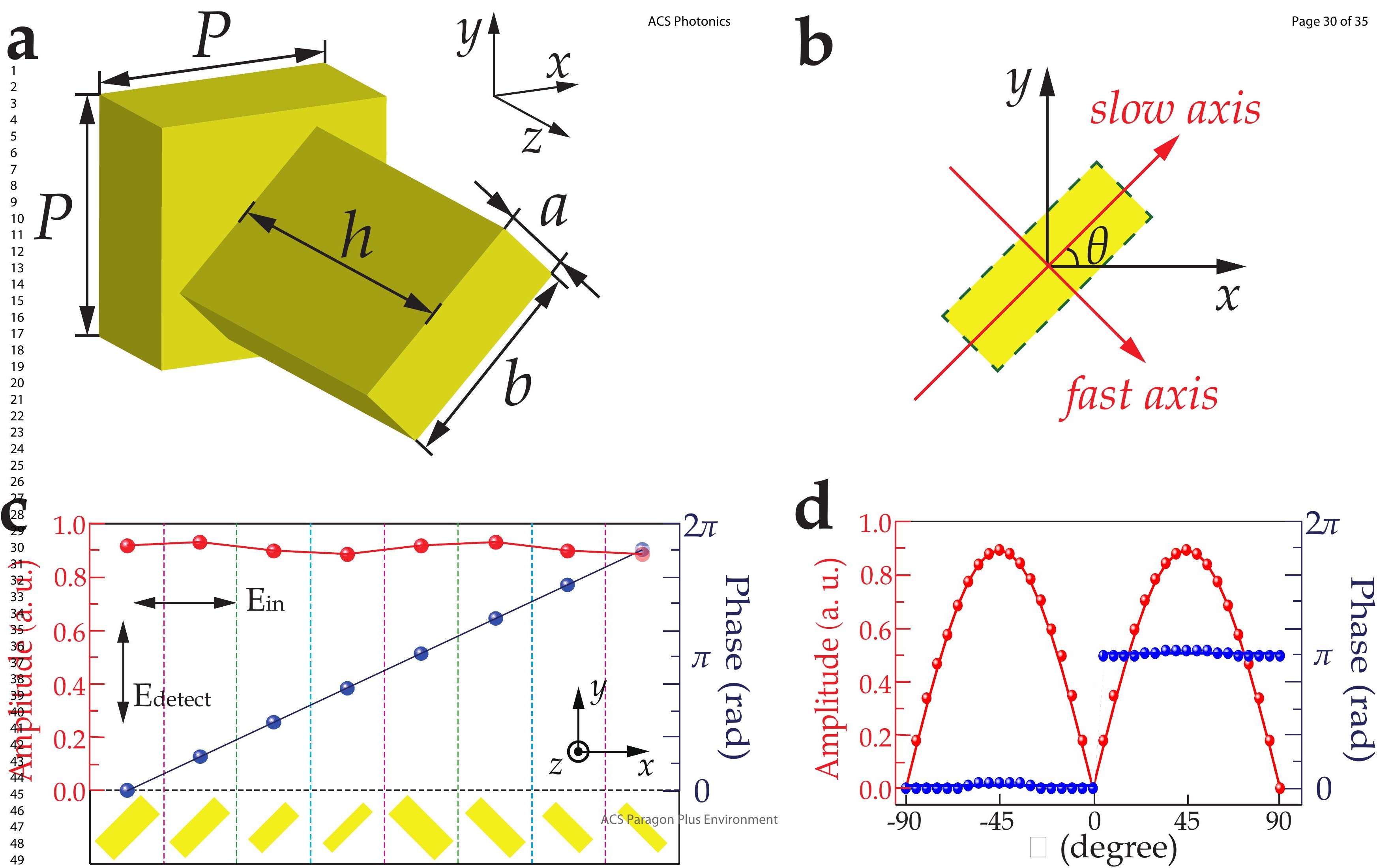




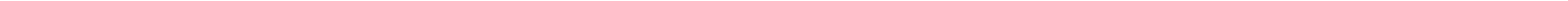




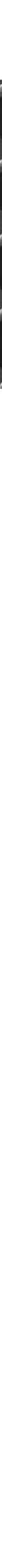




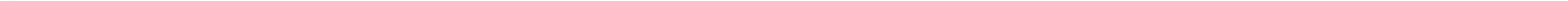




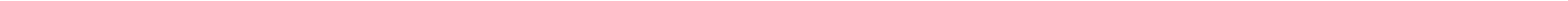

